\title{
Genome-wide identification of cyclin- dependent kinase (CDK) genes affecting adipocyte differentiation in cattle
}

\author{
Cuili Pan ${ }^{1,2+}$, Zhaoxiong Lei ${ }^{1,2+}$, Shuzhe Wang ${ }^{1,2}$, Xingping Wang ${ }^{1,2}$, Dawei Wei ${ }^{1,2}$, Xiaoyan Cai ${ }^{1,2}$, \\ Zhuoma Luoreng ${ }^{1,2}$, Lei Wang ${ }^{3}$ and Yun Ma ${ }^{1,2,3^{*}}$
}

\begin{abstract}
Background: Cyclin-dependent kinases (CDKs) are protein kinases regulating important cellular processes such as cell cycle and transcription. Many CDK genes also play a critical role during adipogenic differentiation, but the role of CDK gene family in regulating bovine adipocyte differentiation has not been studied. Therefore, the present study aims to characterize the CDK gene family in bovine and study their expression pattern during adipocyte differentiation.

Results: We performed a genome-wide analysis and identified a number of CDK genes in several bovine species. The CDK genes were classified into 8 subfamilies through phylogenetic analysis. We found that 25 bovine CDK genes were distributed in 16 different chromosomes. Collinearity analysis revealed that the CDK gene family in Bos taurus is homologous with Bos indicus, Hybrid-Bos taurus, Hybrid Bos indicus, Bos grunniens and Bubalus bubalis. Several CDK genes had higher expression levels in preadipocytes than in differentiated adipocytes, as shown by RNA-seq analysis and qPCR, suggesting a role in the growth of emerging lipid droplets.

Conclusion: In this research, 185 CDK genes were identified and grouped into eight distinct clades in Bovidae, showing extensively homology. Global expression analysis of different bovine tissues and specific expression analysis during adipocytes differentiation revealed CDK4, CDK7, CDK8, CDK9 and CDK14 may be involved in bovine adipocyte differentiation. The results provide a basis for further study to determine the roles of CDK gene family in regulating adipocyte differentiation, which is beneficial for beef quality improvement.
\end{abstract}

Keywords: CDK gene family, Bovine, Adipocytes differentiation, Collinearity analysis, Gene expression pattern

\section{Background}

With the improvement of people's living standards, high-quality beef is becoming more popular. A crucial factor that affects the flavor, freshness, juiciness, tenderness and color in beef is the intramuscular fat (IMF) content. The latter plays an key role in improving taste

\footnotetext{
* Correspondence: mayun_666@126.com

${ }^{+}$Cuili Pan and Zhaoxiong Lei contributed equally to this work.

'School of Agriculture, Ningxia University, Yinchuan 750021, China

${ }^{2}$ Key Laboratory of Ruminant Molecular and Cellular Breeding, Ningxia Hui Autonomous Region, Ningxia University, Yinchuan 750021, China Full list of author information is available at the end of the article
}

and nutritional value, therefore it is important to shed light on the molecular mechanism of IMF deposition in cattle. Adipogenic differentiation is a complex process that includes mitotic clonal expansion (MCE) and terminal adipocyte differentiation [1-3]. Promotion of the cell cycle and maintenance of MCE, necessary for the terminal adipocyte differentiation, depend on cyclindependent kinases [4]. In turn, adipocyte terminal differentiation requires exiting from the mitotic cycle and entry into a permanent state of G1 arrest, together with expression of adipogenic phenotypic genes [1]. PPAR $\gamma$

(c) The Author(s). 2021 Open Access This article is licensed under a Creative Commons Attribution 4.0 International License, which permits use, sharing, adaptation, distribution and reproduction in any medium or format, as long as you give appropriate credit to the original author(s) and the source, provide a link to the Creative Commons licence, and indicate if changes were made. The images or other third party material in this article are included in the article's Creative Commons licence, unless indicated otherwise in a credit line to the material. If material is not included in the article's Creative Commons licence and your intended use is not permitted by statutory regulation or exceeds the permitted use, you will need to obtain permission directly from the copyright holder. To view a copy of this licence, visit http://creativecommons.org/licenses/by/4.0/ The Creative Commons Public Domain Dedication waiver (http://creativecommons.org/publicdomain/zero/1.0/) applies to the data made available in this article, unless otherwise stated in a credit line to the data. 
$\mathrm{C} / \mathrm{EBP} \alpha$ and $\mathrm{C} / \mathrm{EBP} \beta$ are the key transcription factors in the regulatory network that functions during both MCE and terminal adipocyte differentiation [5]. During MCE, $\mathrm{C} / \mathrm{EBP} \beta$ can transactivate the expression of multiple cell cycle-related genes and also facilitate adipocyte hyperplasia by delaying the expression of $C / E B P \alpha$ and $P P A R \gamma$ [5]. When adipocytes accumulate above a threshold, terminal differentiation is initiated, and several genes are expressed to directly or indirectly regulate $C / E B P \alpha$ and $P P A R \gamma$. Then activated PPAR $\gamma$ and C/EBP $\alpha$ regulate the expression of adipogenic phenotypic genes, e.g., FABP1, FABP4, LPL, aP2, CAP and Perilipin, leading to lipid droplet formation [5-7]. Thus, during adipogenic differentiation there is a variety of genes in families such as KLFs, SMADs, RARs, DGATs SREBPS, CDKs and others, that function synergistically $[8,9]$. Herein, we focus on the regulatory mechanism of the CDK gene family in adipocyte differentiation.

CDKs are a large family of serine/threonine protein kinases. They were first discovered in the context of regulation of the cell cycle and have diverse functions in eukaryotes, like mRNA processing, regulation of transcription [10-13]. Recently, they have been shown to regulate adipocyte differentiation and lipid droplet formation by phosphorylating a series of associated transcription factors or adipocyte-specific genes. When the member of the CDK family genes CDK6 was targeted by miR107 in adipocytes, Notch and its downstream gene Hes1 were downregulated and this caused inhibition of glucose uptake and triglyceride synthesis in adipocytes [14]. MAPK and CDK2/cyclinA sequentially activate C/ EBP $\beta$ by maintaining the phosphorylated state of Thr188 during MCE progression and adipocytes terminal differentiation [15]. Insulin activates the CCND3-CDK4 complex which in turn phosphorylates the insulin receptor IRS2 at Ser388; this maintains an active insulin signaling pathway in adipocytes, eventually promoting de novo lipid synthesis [16]. In addition, CDK4 can phosphorylate $\mathrm{Rb}$ to release E2F, leading to preadipocyte proliferation as well as phosphorylation of PPAR $\gamma$ which regulates the terminal differentiation of adipocytes [17]. CDK5 can reduce the insulin sensitivity of adipocytes by phosphorylating PPAR $\gamma$ at Ser273, and inhibition of this phosphorylation promotes browning and thermogenesis in white adipose tissue [18]. The CDK7 complex can inactivate PPARy through PPAR $\gamma$-S112 phosphorylation and inhibit adipogenesis [19]. CDK8 inhibits adipogenesis by phosphorylating the serine residues of SREBP-1c, leading to its ubiquitination and degradation [20]. These findings inspired our curiosity and guided our exploration of the impact of the CDK gene family on bovine adipocyte differentiation.

The expression patterns and regulatory mechanisms of CDK genes in bovine adipocytes have not been systematically studied and elucidated. Therefore, in the present study we aimed to first detect the CDK gene family in the bovine genome and to elucidate their physicochemical properties and structural features together with detailed classification, phylogenetic and functional analyses. In addition, to identify essential members of CDK family that affect adipogenic differentiation, we performed expression pattern analysis by RNA sequencing and qPCR. Our study provides deep insights into CDK genes that influence adipogenic differentiation and is beneficial for future studies to improve IMF in the context of bovine breeding.

\section{Results}

\section{Identification of CDK family members}

To identify the CDK family members, 59 verified CDK amino acid sequences were used as the query. These belonged to cattle (Bos taurus, 8), human (Homo sapiens, 26) and mouse (Mus musculus, 25). Query sequences were used for genome-wide detection of homologous sequences in Bos taurus, Bos indicus, Bos grunniens, $\mathrm{Hy}$ brid-Bos indicus, Hybrid-bos taurus, Bos mutus, Bison bison bison, and Bubalus bubalis. In Bos taurus, 25 nonredundant $C D K$ protein sequences were identified that included CDK1-10, CDK11B, CDK12-20 and CDKL1-5 (Table 1). CDK family proteins were also recognized in Bos grunniens (22), Hybrid-Bos indicus (21), Hybrid-bos taurus (22), Bos mutus (24), Bison bison bison (22), Bos indicus (25) and Bubalus bubalis (24) (Additional file 1). Protein sequences of all CDKs are provided in Additional file 2. A newly identified member of the CDK family was found in Hybrid-bos taurus, Transcript ENSBIXT00000049337, and was named as CDK20 following sequence similarity and collinearity.

The amino acid lengths of the 25 cattle CDK proteins ranged from 292 (CDK5) to 1512 (CDK13), whereas molecular weight $(\mathrm{Mw})$ ranged from $33,288.47$ to 164 , 717.14 $\mathrm{Da}$, consistent with protein length. The isoelectric point $(\mathrm{pI})$ of most CDK family proteins was higher than 8.0, as they contained more basic amino acids than acidic amino acids. However, CDK5 and CDK16 were neutral, with pIs 7.57 and 7.23, respectively. Also, five proteins were acidic (CDK4, CDK6, CDK11B, CDK15 and CDK20) with pIs ranging from 5.34 to 6.68. All 25 CDK proteins contained the Serine/Threonine Kinase (STK) conserved domain (Additional file 3).

\section{Structural features of bovine CDK family members}

To explore the structural characteristics of bovine CDK proteins and genes, the conserved motifs and gene structures were projected based on their phylogenetic relationships (Fig. 1). CDKs of cattle clustered into three main subfamilies according to the evolutionary clades. One subfamily contains 6 members CDKL1-5 and 
Table 1 Characteristics of genome-wide identified CDK family members in Bos taurus

\begin{tabular}{|c|c|c|c|c|c|c|}
\hline Protein Name & Gene ID & Transcript ID & pl & $\mathrm{Mw} / \mathrm{Da}$ & Amino acids & Description \\
\hline CDK1 & ENSBTAG00000010109 & ENSBTAT00000013337 & 8.38 & $34,025.40$ & 297 & cyclin-dependent kinase 1 \\
\hline CDK2 & ENSBTAG00000004021 & ENSBTAT00000005252 & 8.79 & $33,873.46$ & 298 & cyclin-dependent kinase 2 \\
\hline CDK3 & ENSBTAG00000010509 & ENSBTAT00000013885 & 8.13 & $34,805.48$ & 305 & cyclin-dependent kinase 3 \\
\hline CDK4 & ENSBTAG00000007160 & ENSBTAT00000009420 & 6.51 & $33,646.73$ & 303 & cyclin-dependent kinase 4 \\
\hline CDK5 & ENSBTAG00000007766 & ENSBTAT00000010212 & 7.57 & $33,288.47$ & 292 & cyclin-dependent kinase 5 \\
\hline CDK6 & ENSBTAG000000044023 & ENSBTAT00000061349 & 6.22 & $37,014.40$ & 326 & cyclin-dependent kinase 6 \\
\hline CDK7 & ENSBTAG00000011046 & ENSBTAT00000014667 & 8.67 & $38,946.26$ & 346 & cyclin-dependent kinase 7 \\
\hline CDK8 & ENSBTAG00000016737 & ENSBTAT00000022252 & 8.72 & $53,282.71$ & 464 & cyclin-dependent kinase 8 \\
\hline CDK9 & ENSBTAG00000004695 & ENSBTAT00000006162 & 9.04 & $42,747.58$ & 372 & cyclin-dependent kinase 9 \\
\hline CDK10 & ENSBTAG00000033333 & ENSBTAT00000047400 & 9.16 & $41,046.93$ & 361 & cyclin-dependent kinase 10 \\
\hline CDK11B & ENSBTAG00000010737 & ENSBTAT00000014227 & 5.34 & $89,901.85$ & 771 & cyclin-dependent kinase 11B \\
\hline CDK12 & ENSBTAG00000013238 & ENSBTAT00000002005 & 9.54 & $140,641.60$ & 1264 & cyclin-dependent kinase 12 \\
\hline CDK13 & ENSBTAG00000001528 & ENSBTAT00000002003 & 9.71 & $164,717.14$ & 1512 & cyclin-dependent kinase 13 \\
\hline CDK14 & ENSBTAG00000048664 & ENSBTAT00000068321 & 9.06 & $53,169.98$ & 470 & cyclin-dependent kinase 14 \\
\hline CDK15 & ENSBTAG00000055073 & ENSBTAT00000086547 & 6.68 & $45,011.42$ & 405 & cyclin-dependent kinase 15 \\
\hline CDK16 & ENSBTAG00000016769 & ENSBTAT00000022303 & 7.23 & $55,758.68$ & 496 & cyclin-dependent kinase 16 \\
\hline CDK17 & ENSBTAG00000001510 & ENSBTAT00000077282 & 9.1 & $59,563.16$ & 523 & cyclin-dependent kinase 17 \\
\hline CDK18 & ENSBTAG000000012673 & ENSBTAT00000085187 & 9.26 & $54,126.19$ & 471 & cyclin-dependent kinase 18 \\
\hline CDK19 & ENSBTAG00000007288 & ENSBTAT00000009583 & 8.66 & $56,685.13$ & 500 & cyclin-dependent kinase 19 \\
\hline CDK20 & ENSBTAG00000015171 & ENSBTAT00000020188 & 6.06 & $38,546.53$ & 346 & cyclin-dependent kinase 20 \\
\hline CDKL1 & ENSBTAG00000004780 & ENSBTAT00000036046 & 9.08 & $40,735.16$ & 352 & cyclin-dependent kinase like 1 \\
\hline CDKL2 & ENSBTAG00000014038 & ENSBTAT00000031574 & 8.76 & $64,289.09$ & 569 & cyclin-dependent kinase like 2 \\
\hline CDKL3 & ENSBTAG00000010979 & ENSBTAT00000014574 & 9.37 & $67,477.82$ & 591 & cyclin-dependent kinase like 3 \\
\hline CDKL4 & ENSBTAG00000024044 & ENSBTAT00000033135 & 8.88 & $39,465.72$ & 342 & cyclin-dependent kinase like 4 \\
\hline CDKL5 & ENSBTAG000000007428 & ENSBTAT00000076996 & 9.56 & $107,236.16$ & 960 & cyclin-dependent kinase like 5 \\
\hline
\end{tabular}

Mw Molecular weight, $p /$ Isoelectric point. The gene ID and transcript ID can be referred in the Ensembl database (http://asia.ensembl.org/index.html)

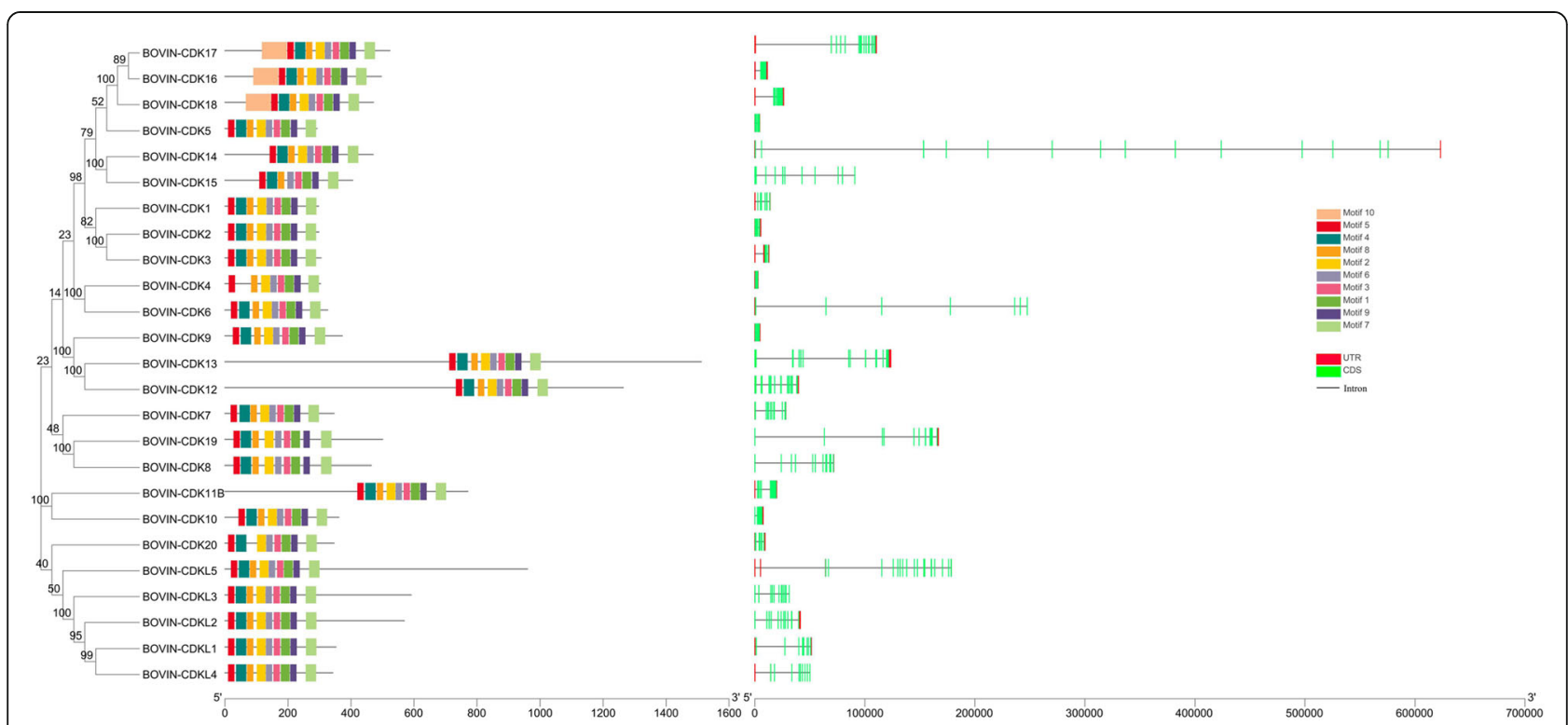

Fig. 1 Characterization of the identified CDK proteins and genes in Bos taurus. The phylogenetic tree (left) was constructed by the Neighbor Joining method. Amino acid sequences (middle), where conserved motifs are indicated as rectangles with different colors. Gene structure map (right), where green rectangle, black line and red rectangle represent CDS, intron and UTR, respectively 
CDK20, another contains CDK10 and CDK11B, whereas the rest belongs to the third subfamily. All the CDK family proteins shared six conserved domains termed motifs $1,3,5,6,7$, and 9, formed by 29, 21, 21, 21, 21 and 21 amino acids, respectively (Additional file 4). A small branch in the third subfamily formed by CDK16, CDK17 and CDK18, has all ten motifs. CDK4, CDK15 and CDK20 all have eight motifs, since CDK4 lacks 4 and 10, CDK15 lacks 2 and 10, and CDK20 lacks 4 and 10. The remaining CDK proteins comprise nine motifs lacking CDK10, which indicates they all have the same conserved patterns.

Introns, coding sequences (CDS) and untranslated regions (UTR) were variable among the CDK gene family. For instance, the length of $\mathrm{CDK}$ genes ranged from $3599 \mathrm{nt}(C D K 4)$ to $678,562 \mathrm{nt}$ (CDK14), mainly due to intron variation. The number of CDS varied from 7 to 17, and the length and layout of 3'UTR and 5'UTR were also variable in the noncoding areas. Despite this variability in CDS, introns and UTRs, we discovered that CDK family members in the same evolutionary branch tend to show similar gene structures and conserved patterns in motifs.

\section{Phylogenetic relationship of CDK proteins in different organisms}

To assess the CDK proteins evolutionary relationships between cattle and other organisms, we conducted a phylogenetic analysis that included eight Bovinae species (Bos taurus, Bos indicus, Bos grunniens, Hybrid-Bos indicus, Hybrid-bos taurus, Bos mutus, Bison bison bison, and Bubalus bubalis) and Homo sapiens and Mus musculus. CDK proteins in human and mouse were also included since they have been studied extensively as model organisms. From these ten organisms, 236 amino acid sequences were aligned to generate a nonrooted Neighbor-Joining (NJ) tree (Fig. 2). Phylogenetic analyses revealed a clustering of $\mathrm{CDK}$ family proteins into eight major clades.

\section{Chromosomal distribution and collinearity analysis of CDK genes}

CDK genes were mapped on the chromosomes of six Bovinae species; 25 bovine CDK genes distribute on 16 chromosomes (Fig. 3). The CDK genes of cattle have a similar distribution as those in Bos indicus. However, the order of CDK14 (7.94-8.62 Mb), CDK6 (9.94-10.19 Mb), CDK13 (80.95-81.08 Mb) and CDK5 (113.630-113.634 $\mathrm{Mb}$ ) in Bos taurus Chr 4 was opposite from that in $\mathrm{Hy}$ brid-Bos indicus, Hybrid-bos taurus and Bos grunniens. In Bos taurus, CDK4, CDK2 and CDK17 were three tandem genes located at $29.66-29.69 \mathrm{Mb}, 29.66-29.69 \mathrm{Mb}$ and 29.72-29.92 Mb on Chr 5, whereas in Bos grunniens, Hybrid-Bos indicus Chr 5 and Bubalus bubalis Chr 4, the arrangement of these three genes was reversed. In addition, compared with Bos taurus, several genes were missing in Bos grunniens (CDK7, CDK11B and CDK20), Hybrid-Bos indicus (CDK11B, CDK16, CDK20 and CDKL4), Hybrid-bos taurus (CDK11B, CDK20, CDKL4 and CDKL5), and Bubalus bubalis $(C D K 11 B)$. Also, CDKL5 is located at chromosome $\mathrm{X}$ in Bos taurus, whereas it is in chromosome Y in Bos grunniens.

Genome collinearity analysis resulted in the identification of pairs of collinear genes between Bos taurus and Bos indicus (31,691), Hybrid-Bos indicus (34,495), Hybrid-bos taurus (33,570), Bos grunniens $(32,378)$ and Bubalus bubalis (33,327), respectively (Fig. 4). There is a one-to-one correspondence between chromosomes of Bos taurus and Hybrid-Bos indicus, Hybrid-bos taurus, Bos indicus and Bos grunniens. A large chromosome homology also existed between cattle $(2 N=60)$ and buffalo $(2 N=50)$, although the chromosome number is different in the two species. The syntenic blocks revealed syntenic relationships in the CDK gene family between cattle and the other five species in Bovinae (Table 2).

\section{Expression analysis of CDK genes in different tissue}

The expression patterns of genes could provide important references for their function. To explore the expression patterns of the CDK gene family during adipogenic differentiation, we investigated the relative expression levels in 163 samples of 60 tissue types. The results showed that CDK genes displayed differential expression patterns in diverse tissues (Fig. 5 a), which could be classified into five groups (A to E). PPARY is a marker gene for adipocyte differentiation, and consistent with this, its expression was high in Group B which included omental, intramuscula, subcutaneous and mammary gland fats. According to their expression patterns, the 25 CDK genes could be grouped into four categories. They were all expressed in the 60 tissues, suggesting a broad regulatory role in life activities. Group I $(C D K 4, C D K 9$ and $C D K 11 B)$ showed the highest expression levels, followed by Group III (CDK3, $C D K 5, C D K 7, C D K 8, C D K 10, C D K 18$, and $C D K 20)$ and Group IV (CDK1, CDK2, CDK6, CDK12, CDK13, CDK14, $C D K 16$, and $C D K 17)$. The rest of the members were in Group II and their expression was the lowest. Further analysis of the five different fat tissues revealed that $C D K 9$ was highly expressed in all of them with an expression pattern similar to PPARY (Fig. 5 b).

\section{Expression analysis of CDK genes in preadipocytes and differentiated adipocytes by RNA-seq}

As revealed by transcriptome analysis, $25 \mathrm{CDK}$ genes showed an up-regulation trend in preadipocytes compared with differentiated adipocytes, except for $C D K 1$, $C D K 3, C D K 6, C D K 19, C D K L 1$ and CDKL4 (Fig. 6). $C D K 7$ displayed high expression, whereas $C D K 1$ showed 


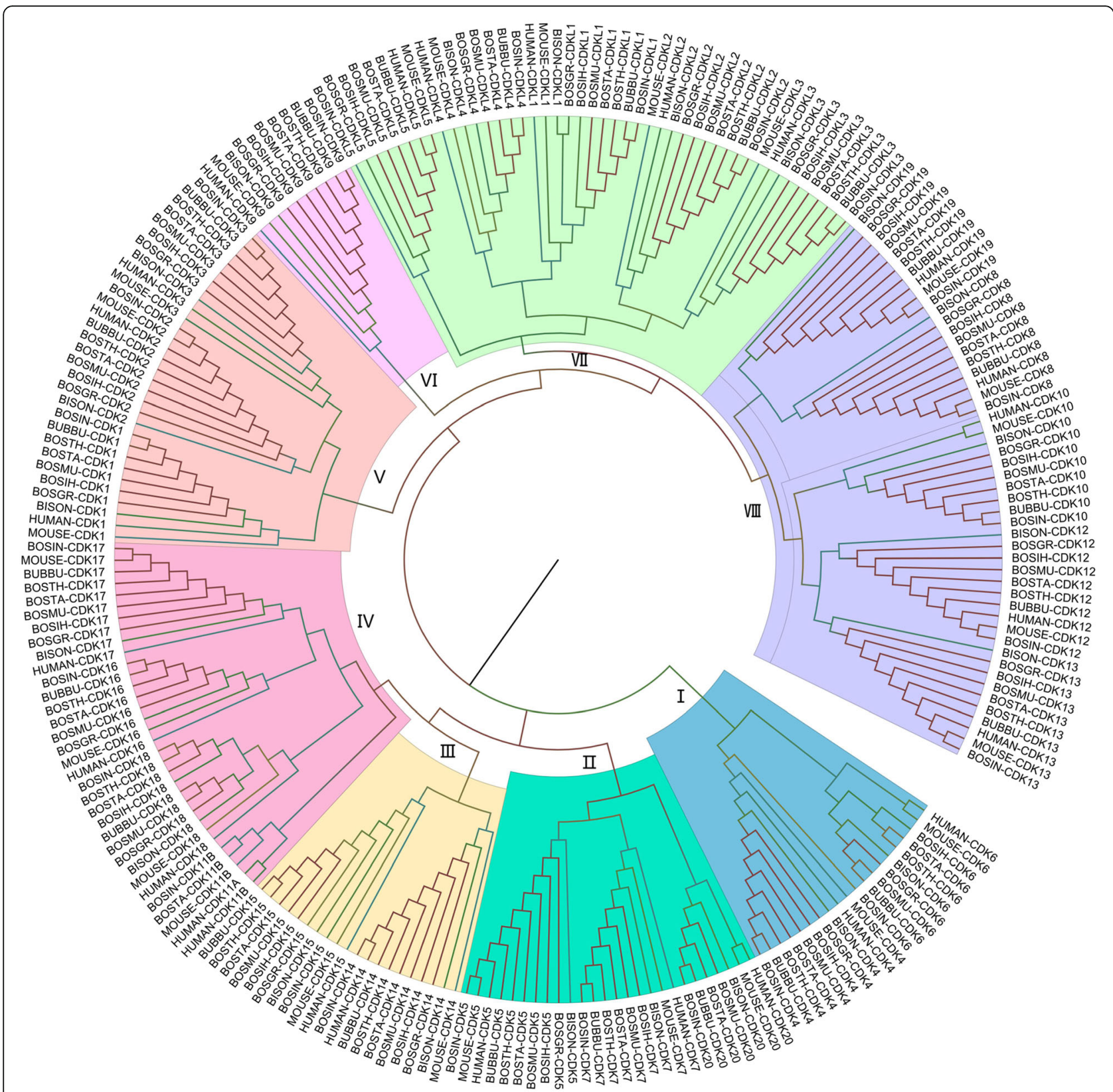

Fig. 2 Phylogenetic Neighbor-Joining (NJ) tree of CDK proteins from ten organisms. Identified CDKs in Bos taurus (BOSTA), BOs grunniens (BOSGR), Hybrid-Bos Indicus (BOSIH), Hybrid-Bos taurus (BOSTH), Bos mutus (BOSMU), Bison bison bison (BISOM), Bos indicus (BOSIN) and Bubalus bubalis (BUBBU) together with verified CDKs from Homo sapiens (HUMAN) and Mus musculus (MOUSE). CDK proteins are grouped into eight clusters (I-VII) shown as different colors

low expression in preadipocytes (within the $95 \%$ confidence interval). CDK4, CDK8, CDK9 and CDK14 displayed a high expression in preadipocytes (within the $99 \%$ confidence interval).

\section{Expression analysis of CDK genes during adipocyte differentiation by $\mathrm{qPCR}$}

To further explore the expression patterns of the CDK gene family, preadipocytes collected from perirenal adipose tissue of premature calves were induced to differentiate. The results of Oil Red O staining showed that lipid droplet accumulation increased in adipocytes induced for 10 days compared to preadipocytes (Additional file 5), indicating that induction and differentiation was successful. A qPCR analysis was conducted to detect the expression of CDK genes at $0,2,4$, 6 , and 10 days during adipocytes differentiation (Fig. 7). CDK genes showed a relatively high expression in 


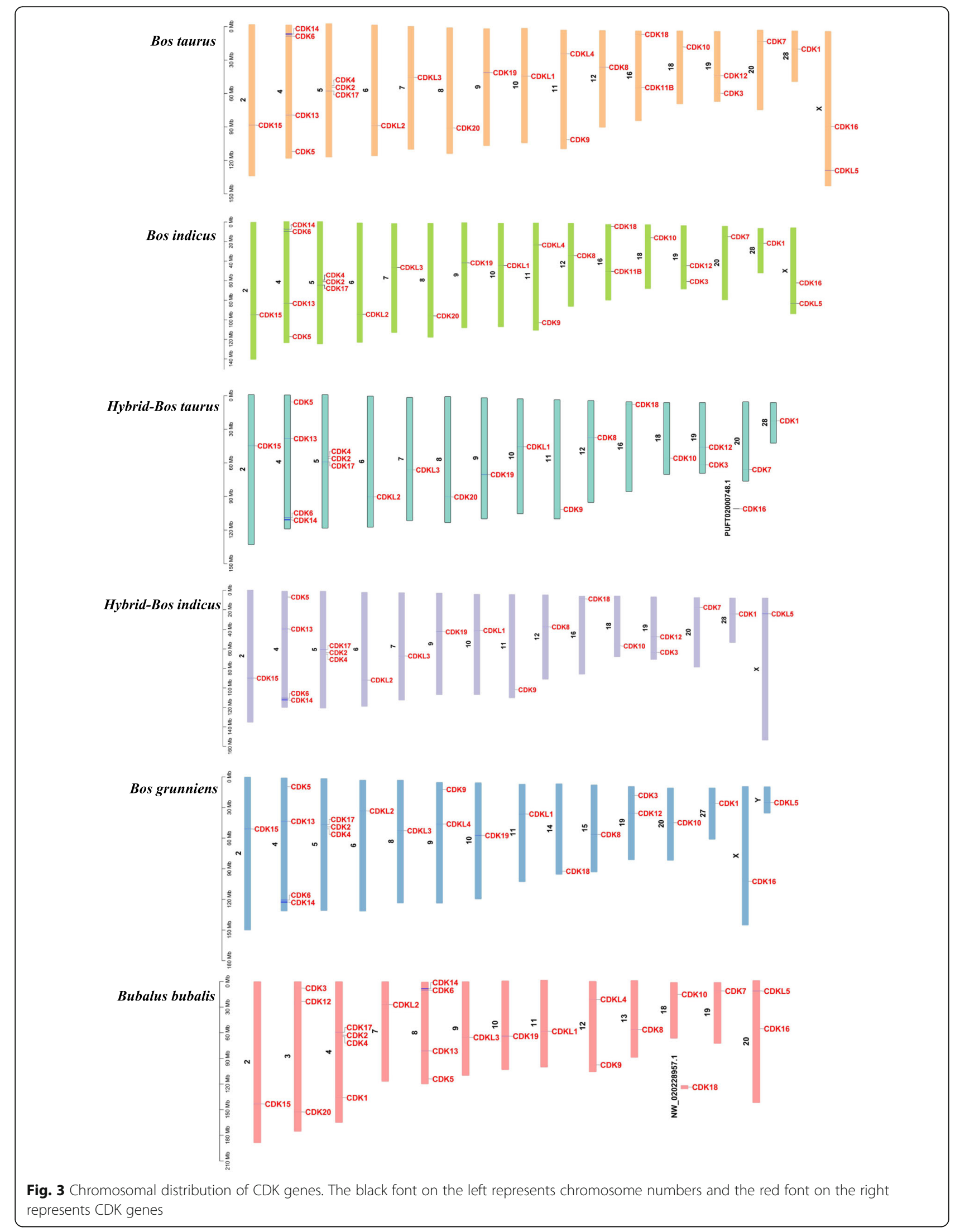




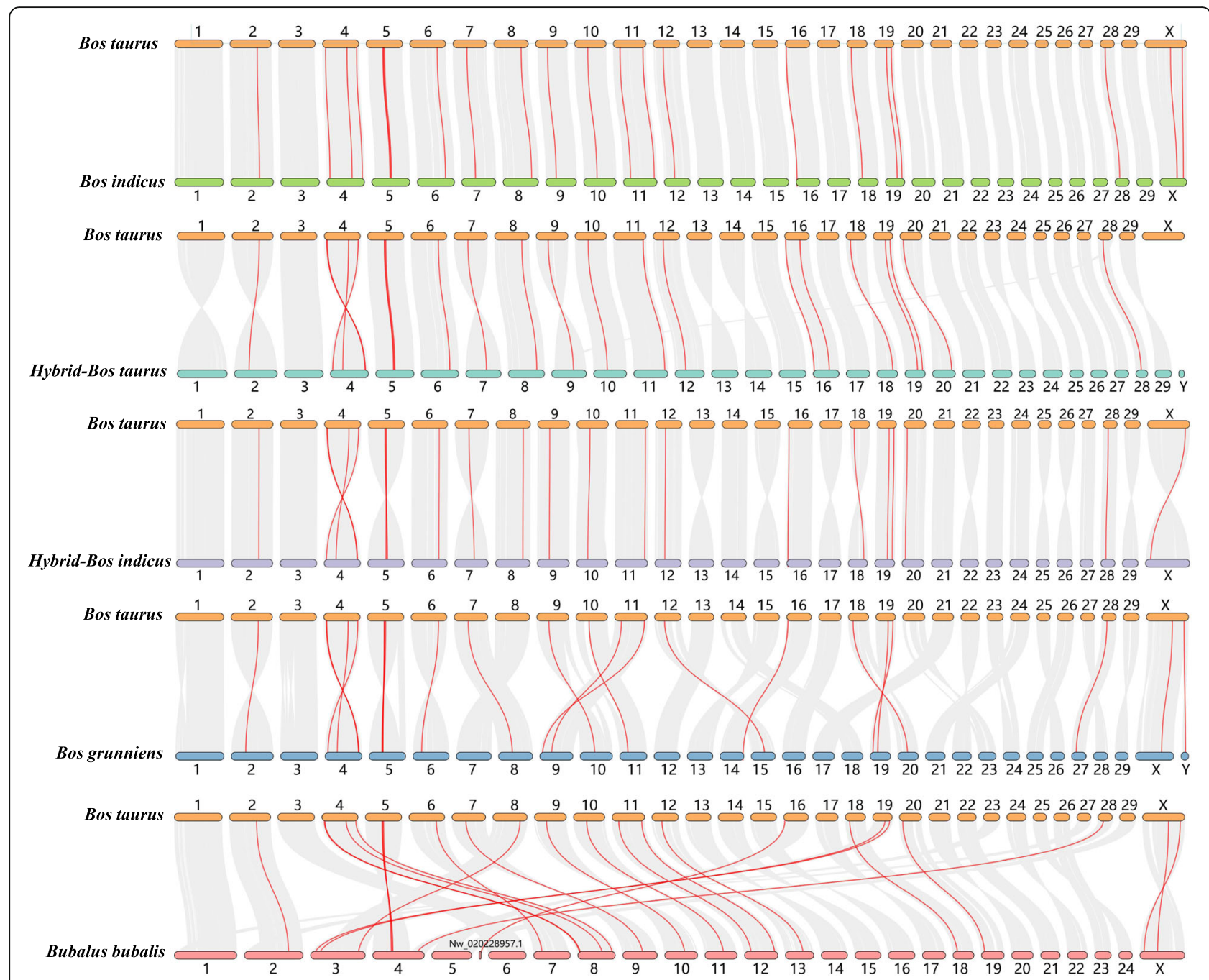

Fig. 4 Collinearity analysis of CDK genes between cattle and other organisms. Syntenic genes pairs are linked by grey lines whereas syntenic CDK genes are shown as red lines

preadipocytes and then decreased as differentiation progressed in addition to CDK1, CDK15, CDK18, CDKL3 and CDKL5. CDK1,CDKL3 and CDKL5 showed the highest expression on the second day of differentiation whereas the lowest expression points were on the 6th, 8th and 6th day, respectively. CDK15 and CDK18 increased with adipocyte differentiation and reached a peak on the fourth day, then decreased.

\section{Discussion}

Cattle is known as an important species for supplying meat. The IMF content directly affects the taste and flavor of beef and it is of great scientific significance to reveal the molecular regulation mechanism of IMF deposition. The CDK gene family encoding functional proteins have been well studied in the regulation of transcription, metabolism and cell differentiation [10-12]. However, research on the role of CDK genes in adipocyte differentiation is limited, especially in Bovidae. Since cattle and several species of Bovidae were sequenced, these resources may serve as reference to explore the evolution and function of CDK genes and in advancing genome science in Bovidae.

\section{Structural features of bovine CDK family proteins and genes}

The activity of proteins depends on their functional motifs and domains [21]. Six conserved amino acid sequences (Motifs 1, 3, 5, 6, 7 and 9) were conserved among all CDK family members in cattle. Highly conserved motifs are usually located in active sites of enzymes and are essential in maintaining their structure, binding to substrate or in catalysis [22, 23]. CDK16-18, a small branch in a subfamily, have all of the ten motifs, pointing to a common function in these three members. It is likely that Motif 10 is not located at the core of the 
Table 2 Syntenic relationships of CDK family genes between cattle and the other five species

\begin{tabular}{|c|c|c|c|c|c|}
\hline Gene & Bos indicus & Hybrid-bos taurus & Hybrid-Bos Indicus & Bos grunniens & Bubalus bubalis \\
\hline CDK1 & $Y$ & Y & Y & $Y$ & $Y$ \\
\hline CDK2 & Y & Y & Y & Y & Y \\
\hline CDK3 & Y & Y & Y & Y & Y \\
\hline CDK4 & Y & Y & Y & Y & Y \\
\hline CDK5 & Y & Y & Y & Y & Y \\
\hline CDK6 & Y & Y & Y & Y & Y \\
\hline CDK7 & $\mathrm{N}$ & Y & Y & - & Y \\
\hline CDK8 & Y & Y & Y & Y & Y \\
\hline CDK9 & Y & Y & Y & Y & Y \\
\hline CDK10 & Y & Y & Y & Y & Y \\
\hline CDK11B & $\mathrm{N}$ & - & - & - & - \\
\hline CDK12 & Y & Y & Y & Y & Y \\
\hline CDK13 & Y & Y & Y & Y & Y \\
\hline CDK14 & Y & Y & Y & Y & Y \\
\hline CDK15 & Y & Y & Y & Y & Y \\
\hline CDK16 & Y & Y & - & Y & Y \\
\hline CDK17 & Y & Y & Y & Y & Y \\
\hline CDK18 & Y & Y & $Y$ & Y & $Y$ \\
\hline CDK19 & Y & Y & $Y$ & Y & Y \\
\hline CDK2O & Y & Y & - & - & $Y$ \\
\hline CDKL1 & Y & Y & $Y$ & Y & $Y$ \\
\hline CDKL2 & Y & Y & Y & Y & Y \\
\hline CDKL3 & Y & Y & Y & Y & Y \\
\hline CDKL4 & Y & - & - & Y & Y \\
\hline CDKL5 & Y & - & $Y$ & Y & $Y$ \\
\hline
\end{tabular}

' $\mathrm{Y}$ ' represents the synteny of genes between two species, while ' $\mathrm{N}$ ' means not and '-' means lacking of the gene

catalytic domain since the other 22 members where it is absent still show kinase activities. In addition to Motif 10, other motifs are absent in CDK4 (Motif 4), CDK15 (Motif 2) and CDK20 (Motif 8) indicating some sequence losses during evolution. CDK20 is a newly identified member in Hybrid-bos taurus, and has a conserved STKc domain and nine motifs (Additional file 6). Domain and motif analysis revealed its similarity with the other CDK proteins.

The distribution of CDSs, introns, and UTRs was variable in CDK genes, mainly due to the length and layout of introns and UTRs, whereas gene coding sequences and amino acid sequences were similar. These results suggest that amino acid sequence similarity, especially in conserved motifs, may play essential roles in keeping the kinase function in CDK proteins.

\section{Phylogenetic relationship of CDK family proteins}

Phylogenetic analysis of proteins can provide an indeep insight for their evolutionary relationships [24]. CDK family proteins were classified into eight major clades. The same member from each species first clustered in one branch, indicating that they were conserved in sequences among the ten species. Initially, Clade I separated, whereas Clade II, III and IV clustered into a subfamily and the remaining clustered into another subfamily. This shows that they have evolved asymmetrically and that these subfamilies are distant. Notably, Clade VII includes all the cyclin-dependent kinases like proteins (CDKL1, CDKL2, CDKL3, CDKL4 and CDKL5), consistent with human study that divided the CDK gene family into CDK and CDKL [12]. As expected, members that are closer evolutionarily clustered together first. For example, Bos indicus CDK1 first clustered with that of Bubalus bubalis, and then get together with Hybridbos taurus, Bos taurus, Bos mutus, Hybrid-Bos indicus, Bos grunniens, Bison, human and mouse.

\section{Collinearity analysis of CDKs in Bovidae}

Members of a gene family may distribute in the same or in different chromosomes. These two cases are due to 


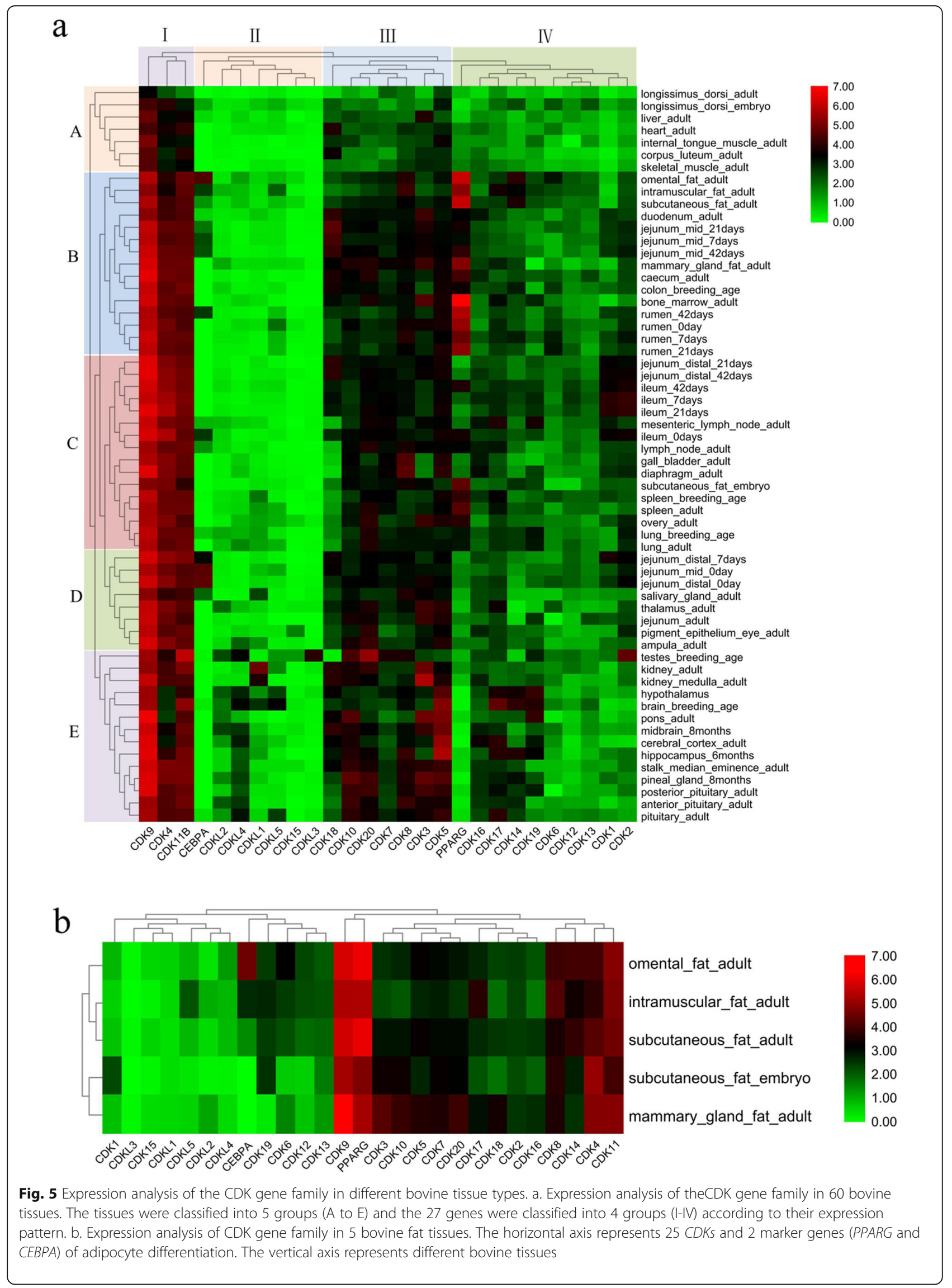




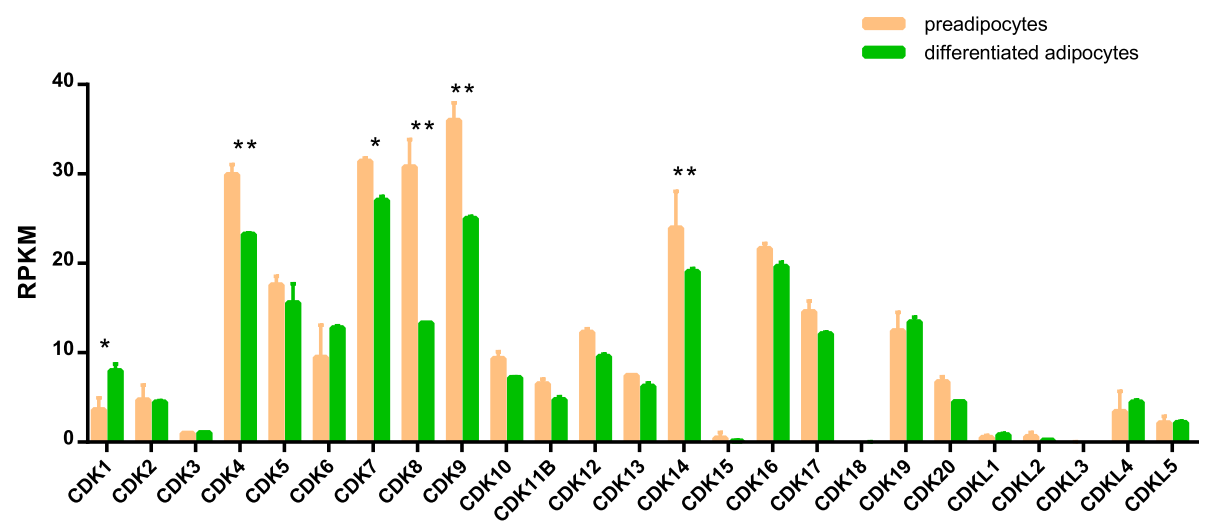

Fig. 6 Expression analysis of CDK genes in preadipocytes and differentiated adipocytes by RNA-seq. Error bars were obtained from two measurements. Symbols * and ** above the bars indicate significant differences at 0.05 and 0.01 level between preadipocytes and differentiated adipocytes

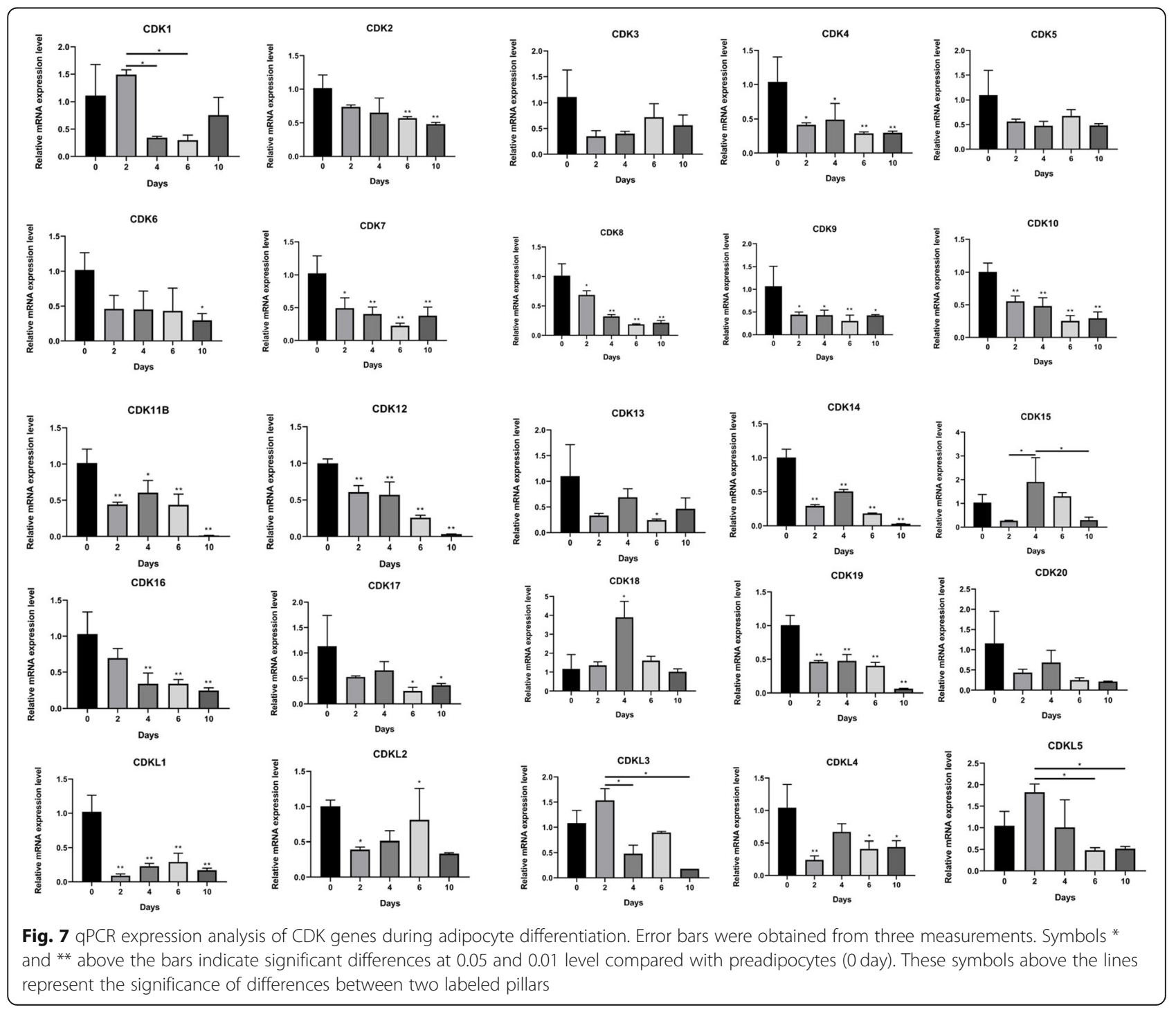


tandem or segmental duplication events, respectively [25-27]. The members of the CDK gene family showed a distribution in 13 to 16 chromosomes in six species of Bovidae, indicating that both segmental and tandem duplication events have occurred. In addition to a pair sex chromosomes (XX/XY), the genomes of Bos taurus, Bos grunniens, Hybrid-Bos indicus, Hybrid-bos taurus, and Bos indicus consist of 29 autosomes [28, 29], whereas Bubalus bubalis has 24 autosomes. The collinearity results showed a one-to-one correspondence between chromosomes of Bos taurus and Hybrid-Bos indicus, Hybrid-bos taurus, Bos indicus and Bos grunniens, and large homologous chromosomal regions between Bos taurus and Bubalus bubalis. The arrangement of genes in some syntenic chromosomes may be totally reversed between Bos taurus and other species. This may be due to the opposite starting point for chromosome annotation. Since all the chromosomes were homologous in Bovidae, the positions where CDK genes located were either collinear (conserved in the same order) or syntenic (not necessarily in the same order) between each two species except for only a few gene pairs [30]. For example, $C D K 7$ in Chr 20 and CDK11B in Chr 16 did not show synteny between Bos taurus and Bos indicus. The positions of CDKL4 and CDK9 are opposite between Bos taurus and Bos grunniens, which located at $21.50-21.55 \mathrm{Mb}$ and 98.46-98.47 Mb in Chr 11 of Bos taurus and 41.0241.08 Mb and 7.13-7.14 Mb in Chr 9 of Bos grunniens. The deficiency and discrepancies of CDK genes might be caused by the sequence variation and chromosome rearrangement in the process of evolution [31]. In addition, Chr16 of Bos taurus showed a syntenic relationship with Chr5 and scaffold NW_020228957.1 of Bubalus bubalis, suggesting that NW_020228957.1, which has not been assembled yet, may be a part of Bubalus bubalis Chr5. Meanwhile, it was syntenic between CDK18 of Bos taurus Chr16 and that in scaffold NW_020228957.1 of Bubalus bubalis. In a word, the extensive homology provided rich perspectives for studying the function and evolution of CDK gene family in Bovidae.

\section{CDK genes affecting adipocyte differentiation}

CDK family proteins can regulate adipocyte differentiation by phosphorylating a series of transcriptionrelated factors or adipocyte-specific genes [14-18, 20]. To dissect the expression pattern of this family, the expression of its 25 members in 60 tissue types was analyzed. $C D K 4, C D K 9$ and $C D K 11 B$ were highly expressed in four types of fat tissues (omental, intramuscular, subcutaneous and mammary gland) relative to other tissues. Thus, we hypothesized that these three genes may be more relevant to adipose tissue regulation than other members. Indeed, CDK4 can phosphorylate IRS2 and $\mathrm{Rb}$ to promote adipogenesis [16, 17], whereas CDK9, a component of positive transcription elongation factor $b(\mathrm{P}-\mathrm{TEFb})$, can phosphorylate the C-terminal domain of RNA polymerase II and regulate the transcription of target genes by facilitating transcriptional elongation [32]. In $3 \mathrm{~T} 3-\mathrm{L} 1$ cells, CDK9 increased the adipogenic potential by phosphorylating PPARY directly and inducing its transcriptional activity [33]. In contrast, although $C D K 11 B$ had similar expression patterns to $C D K 4$ and $C D K 9$, its role in adipogenic differentiation has never been described.

Adipogenic differentiation is a complicated and wellorganized process regulated by multiple genes. Analysis of CDK gene family expression patterns during adipocyte differentiation is essential to explore its role. CDK4, $C D K 7, C D K 8$ and $C D K 9$ were highly expressed in preadipocytes, suggesting a role in targeting newly generated lipid droplets. CDK1,CDKL3 and CDKL5 reached the highest expression on the second day, whereas $C D K 15$ and $C D K 18$ peaked on the fourth day, indicating that they may also play regulatory roles during adipocyte differentiation. The role of CDK1, CDK4, CDK7, CDK8 and $C D K 9$ in adipocyte differentiation has been studied $[16,17,19,20,33-37]$ but they need to be explored in more detail, whereas CDK15, CDK18, CDKL3 and $C D K L 5$ have not been studied.

Interestingly, the expression trends of some of the members were inconsistent between RNA-seq and qPCR validation tests. For instance, RNA-seq analysis showed no significant differences in the expression of $C D K 2$, CDK6, CDK10, CDK11B, CDK12, CDK16, CDK17, $C D K 19$ and $C D K L 1$, but qPCR showed significant downregulation. This may have been caused by an insufficient number of samples and/or the different sample source used for qPCR. RNA-seq samples were separated from inguinal subcutaneous fat of two 1 year old male Qinchuan cattles, whereas qPCR samples were from perirenal fat of a premature female Holstein calf. Thus, the determination of the function of CDK genes during adipocyte differentiation is complicated and requires an in-depth analysis.

The CDK gene family and the interacting genes formed an integrated network as shown by literature mining using the Agilent Literature Search plug-in of Cytoscape (Additional file 7) [38]. For example, CDK7 could directly activate $C D K 9$ to maintain a high expression of MDM4 and MDM2 [36, 37]. MDM2 facilitates adipocyte differentiation through CRTC-mediated activation of STAT3 [39]. Overall, these results revealed that the CDK gene family interact with each other and other genes, playing non-redundant roles and collectively regulating cell cycle, adipocyte differentiation or lipid metabolism. 


\section{Conclusions}

We have conducted a comprehensive genome-wide analysis of the CDK gene family in Bovidae. A total of 185 CDK genes were identified and grouped into eight distinct clades. Collinearity analysis revealed that the CDK gene family is homologous between cattle and other species in Bovinae. Expression analysis and functional prediction indicated that CDK genes may play a significant and complicated role in regulating bovine adipocyte differentiation. These results provide an essential reference for further studies of the CDK gene family in the regulation of bovine adipocyte differentiation.

\section{Methods}

\section{Ethics statement}

Animal experiments were conducted according to the guidelines of the Regulations for the Administration of Affairs Concerning Experimental Animals (Ministry of Science and Technology, China, 2004). All animal protocols were approved by the Animal Ethics Committee of Ningxia University (permit number NXUC20200618) and Zerui ecological breeding farm (permit number ZR20200615). A premature female calf of a Holstein pregnant cow used in the experiment was released and the primary adipocytes were isolated immediately, making all efforts to minimize suffering of the calf. The pregnant cow was not sampled and is still being raised in Zerui ecological breeding farm (Yinchuan, China) after a period of recuperation.

\section{Genome-wide identification of CDK genes}

The genome and annotation of Bos taurus (ARSUCD1.2 assembly), Bos grunniens (LU_Bosgru_v3.0 assembly), Hybrid-Bos indicus (Bos indicus $\times$ Bos taurus, UOA_Brahman_1 assembly), Hybrid-bos taurus (Bos indicus $\times$ Bos taurus, UOA_Angus_1 assembly), Bos mutus (BosGru_v2.0 assembly), Bison bison bison (Bison_UMD1.0 assembly), Homo sapiens (GRCh38.p13 assembly) and Mus musculus (GRCm39 assembly) are from Ensembl database (http://useast.ensembl.org/info/ data/ftp/index.html); Bos indicus (Bos_indicus_1.0 assembly) and Bubalus bubalis (UOA_WB_1 assembly) are from NCBI Genome repository (https://www.ncbi. nlm.nih.gov/genome/). To identify all the possible CDK genes in Bovinae, both Hidden Markov Model (HMM) search and Basic Local Alignment Search Tool (BLAST) were performed [40]. A total of 59 reviewed CDKs sequences of bovine (Bos taurus), human (Homo sapiens) and mouse (Mus musculus) were obtained from the UniProt database (https://www.uniprot.org/). These protein sequences were used as seeds to query potential CDK gene family candidates via BLASTP with a threshold of e-value $=10^{-5}$. The HMM of CDKs (PF00069) was downloaded from Pfam (https://pfam.xfam.org/) [41] and HMMER 3.3.1 (http://hmmer.org/) [42] was used to construct HMM profiles in Bovidae for detection of CDK genes with the default setting. Candidate sequences obtained from the two methods were manually checked to confirm the CDK homology. Non-redundant CDK homologs were submitted to NCBI CD-search [43] to verify the presence of the conserved protein domains. Molecular weight and isoelectric point were calculated by ExPASy (https://web.expasy.org/protparam/) [44].

\section{Phylogenetic analysis}

The known CDK amino acid sequences in the Homo sapiens and Mus musculus were downloaded from the UniProt database (https://www.uniprot.org/) (Additional file 2). These, together with the identified and known amino acid sequences of CDKs in Bos taurus, Bos grunniens, Hybrid-Bos indicus, Hybrid-bos taurus, Bos mutus, Bison bison bison, Bos indicus and Bubalus bubalis, were aligned by ClustalW and a Neighbor-Joining tree was constructed in MEGA 7.0 [45]. The bootstrap was set as 1000 replicates. FigTree software (version 1.4.3) was used to adjust and beautify the evolutionary tree.

\section{Structural features analysis}

To further evaluate the structural diversity of cattle CDK genes and proteins, a phylogenetic Neighbor-Joining tree was constructed. The conserved motifs were detected in MEME 5.0 [46] and visualized in TBtools [47]. The minimum and maximum number of amino acids in each motif was 6 and 50, respectively. The number of motifs in each CDK protein was limited to 10 . Coding sequences and corresponding genomic sequences of bovine CDK genes were loaded into TBtools to portray the number and positions of CDSs and introns graphically.

\section{Chromosomal distribution and collinearity analysis}

Positional information of predicted CDK genes in Bos taurus, Bos grunniens, Hybrid-Bos indicus, Hybrid-bos taurus, Bos indicus and Bubalus bubalis was extracted from the genomic sequence and annotation files and then was visualized in TBtools [47]. The identified CDKs in each species were mapped onto chromosomes. Comparisons between pairs of genomes were performed by all-against-all BLASTP searches (e-value $=10^{-5}$ ) using the proteome sequences of Bos taurus as queries against those of the other five bovine species above. Collinearity analysis of ortholog genes between Bos taurus and the other five species was conducted using the MCScanX toolkit [48]. Results of collinearity analysis and orthologous CDKs were visualized by TBtools [47].

\section{Gene expression analysis by RNA-seq}

RNA-Seq data of preadipocytes and differentiated adipocytes was downloaded from the National Center for 
Biotechnology Information (NCBI) Sequence Read Archive (accession number SRP067820) [49] and transformed into fastaq format by Fastq-dump. The sequencing quality was checked using FastQC [50]. Quality control of raw sequence data, including removal of the adapter sequences and low-quality sequences was performed using the Trim_galore [51]. Clean reads were then mapped to the Bos taurus genome (ARS-UCD1.2.101) using STAR [52]. RSEM [53] and FeatureCounts [54] were used to calculate the expression of the transcripts. Data was normalized by calculating the RPKM for each gene. The limma package [55] in $\mathrm{R}$ was used to analyze the differential expression under the threshold of fold change $\geq 1.2$ and $p$-value $<0.05$. The Sequence Read Archive Run (SRR) number and adjusted RPKM values of 163 tissue samples were downloaded from the Ruminant Genome Database (http://animal.nwsuaf.edu.cn/code/ index.php/Ruminantia) [56] (Additional file 8). The heat map was constructed using the pheatmap package in $\mathrm{R}$ software [57].

\section{Isolation, culture and induction differentiation of bovine primary adipocytes}

Primary adipocytes were isolated and cultured from perirenal adipose tissue of premature calf in the Zerui ecological breeding farm. Type I collagenase digestion method was used for the isolation and cultivation of calf preadipocytes. Induction of preadipocytes differentiation [58] and Oil Red O staining [59] were performed as described. Images were captured using a $10 \times$ objective lenses without filter model on an OLYMPUS IX50 microscope (Olympus Corporation, Tokyo, Japan) equipped with a charge-coupled device (CCD) camera (Diagnostic Institute, Inc., Sterling Height, MI, USA). CellSens V3.2 acquisition software was used and the images with a resolution at $96 \mathrm{dpi}$ were acquired.

\section{RNA extraction and quantitative RT-PCR (qPCR)}

The primers of the CDK genes were designed using Primer Premier 5.0 software (Additional file 9). Total RNA was extracted by the phenol-chloroform method using TRIzol (9109, Takara), and bovine adipocytes $(n=3)$ during the differentiation of $0,2,4,6$ and 10 days were used. Absorbance was measured at $260 \mathrm{~nm}$ and $280 \mathrm{~nm}$ and samples with an OD260/OD280 ratio between 1.8 and 2.0 were used in subsequent experiments. Then, 1000 ng total RNA was reverse transcribed using random primers with Moloney murine leukemia virus reverse transcriptase (Takara Bio, Kyoto, Japan). Realtime PCR was carried out in a CFX96 Touch Real-Time PCR Detection System (Bio-Rad, Hercules, CA, USA) with SYBR Green Master Mix (Takara Bio, Kyoto, Japan). The qPCR reaction procedure was 40 cycles of predenaturation for $3 \mathrm{~min}\left(95^{\circ} \mathrm{C}\right)$, denaturation for $10 \mathrm{~s}$ $\left(95^{\circ} \mathrm{C}\right)$, annealing for $20 \mathrm{~s}\left(59-61^{\circ} \mathrm{C}\right)$, extension for $30 \mathrm{~s}$ $\left(72^{\circ} \mathrm{C}\right)$. Glyceraldehyde-3-phosphate dehydrogenase (GAPDH) was selected as the reference gene.

\section{Statistical analysis}

All qRT-PCR results were determined using the $2^{-\Delta \Delta C t}$ method [60, 61]. Three independent technical repetitions were performed for each test. Statistical significance determined using Graphpad Prism 7.0 software.

\section{Abbreviations}

CDK: Cyclin-dependent kinases; MCE: Mitotic clonal expansion; CDS: Coding sequences; HMM: Hidden Markov Model; Chr: Chromosome;

IMF: Intramuscular fat; pl: Isoelectric points; Mw: Molecular weight

\section{Supplementary Information}

The online version contains supplementary material available at https://doi. org/10.1186/s12864-021-07653-8.

Additional file 1. Genome-wide identified CDK family members in Bovidae.

Additional file 2. Protein sequences of CDK family members in ten species.

Additional file 3. Conserved domain prediction of bovine CDK protein sequences.

Additional file 4. Amino acid sequences logos of 10 identified motifs in bovine CDK proteins.

Additional file 5. Oil Red O staining of preadipocytes and differentiated adipocytes.

Additional file 6 Conserved domain and motifs of hybrid-Bos taurus CDK proteins.

Additional file 7. The interaction network for CDK genes constructed by Cytoscape.

Additional file 8. Expression values (RPKM) of 163 bovine samples in 60 tissue types.

Additional file 9. Primers sequences of 25 bovine CDK genes.

\section{Acknowledgments}

We would like to express our gratitude to EditSprings (https://www. editsprings.com/) for the expert linguistic services provided. We would also like to thank Qingfeng Li and Chengjie Chen for providing valuable advice in the research.

\section{Authors' contributions}

CLP and ZXL made the same contribution to the study. Conceived and designed the research: YM and CLP; Analyzed the data and conducted the experiment: CLP and ZXL; Wrote the paper: CLP; Modified manuscript: YM, ZXL, SZW, XPW, DWW, XYC, LW, ZMLR. All authors read and approved the final manuscript.

\section{Funding}

This study was funded by the National Natural Science Foundation of China (32072720 and 31672403), the Leading Talents Fund in Science and Technology Innovation in Henan Province (No. 194200510022), the Key Research and Talent Introduction Project of Ningxia Hui Autonomous Region (2019YCZX0068) and the Science and Technology Innovation Team Projects of Ningxia Hui Autonomous Region (03010360052). The funding bodies played no role in the design of the study, collection, analysis, and interpretation of data and writing the manuscript.

Availability of data and materials

The datasets analysed during the current study are available in the NCBI Short Read Archive (SRA) repository, [accession number SRP067820; web links for SRP067820: https://trace.ncbi.n/m.nih.gov/Traces/study/?acc=SRP06 
7820\&o=acc_s\%3Aa; direct web links for SRR3056892 (https://sra-downloadb. be-md.ncbi.nlm.nih.gov/sos2/sra-pub-run-7/SRR3056892/SRR3056892.1), SRR3064490 (https://sra-downloadb.be-md.ncbi.nlm.nih.gov/sos2/sra-pubrun-7/SRR3064490/SRR3064490.1), SRR3064491 (https://sra-downloadb.be-md. ncbi.nlm.nih.gov/sos2/sra-pub-run-7/SRR3064491/SRR3064491.1), and SRR3064492 (https://sra-downloadb.be-md.ncbi.nlm.nih.gov/sos2/sra-pubrun-7/SRR3064492/SRR3064492.1)].

The web links for genome and annotation of Bos taurus are available in http://ftp.ensembl.org/pub/release-103/fasta/bos_taurus/dna/Bos_taurus.ARSUCD1.2.dna.toplevel.fa.gz and http://ftp.ensembl.org/pub/release-103/gff3/ bos_taurus/Bos_taurus.ARS-UCD1.2.103.gff3.gz;

the web links for genome and annotation of Bos grunniens are available in http://ftp.ensembl.org/pub/release-103/fasta/bos_grunniens/dna/Bos_ grunniens.LU_Bosgru_v3.0.dna.toplevel.fa.gz and http://ftp.ensembl.org/pub/ release-103/gff3/bos_grunniens/Bos_grunniens.LU_Bosgru_v3.0.103.gff3.gz; the web links for genome and annotation of Hybrid-bos taurus are available in http://ftp.ensembl.org/pub/release-103/fasta/bos_taurus_hybrid/dna/Bos_ taurus_hybrid.UOA_Angus_1.dna.toplevel.fa.gz and http://ftp.ensembl.org/ pub/release-103/gff3/bos_taurus_hybrid/Bos_taurus_hybrid.UOA_Angus_1.1 03.gff3.gz:

the web links for genome and annotation of Hybrid-Bos indicus are available in http://ftp.ensembl.org/pub/release-103/fasta/bos_indicus_hybrid/dna/Bos_ indicus_hybrid.UOA_Brahman_1.dna.toplevel.fa.gz and http://ftp.ensembl. org/pub/release-103/gff3/bos_indicus_hybrid/Bos_indicus_hybrid.UOA_ Brahman_1.103.gff3.gz;

the web links for genome and annotation of Bos mutus are available in http://ftp.ensembl.org/pub/release-103/fasta/bos_mutus/dna/Bos_mutus. BosGru_v2.0.dna.toplevel.fa.gz and http://ftp.ensembl.org/pub/release-103/ gff3/bos_mutus/Bos_mutus.BosGru_v2.0.103.gff3.gz;

the web links for genome and annotation of Bison bison bison are available in http://ftp.ensembl.org/pub/release-103/fasta/bison_bison_bison/dna/ Bison_bison_bison.Bison_UMD1.0.dna.toplevel.fa.gz and http://ftp.ensembl. org/pub/release-103/gff3/bison_bison_bison/Bison_bison_bison.Bison_ UMD1.0.103.gff3.gz;

the web links for genome and annotation of Homo sapiens are available in http://ftp.ensembl.org/pub/release-103/fasta/homo_sapiens/dna/Homo_ sapiens.GRCh38.dna.toplevel.fa.gz and http://ftp.ensembl.org/pub/release-1 03/gff3/homo_sapiens/Homo_sapiens.GRCh38.103.gff3.gz;

the web links for genome and annotation of Mus musculus are available in http://ftp.ensembl.org/pub/release-103/fasta/mus_musculus/dna/Mus_ musculus.GRCm39.dna.toplevel.fa.gz and http://ftp.ensembl.org/pub/release-1 03/gff3/mus_musculus/Mus_musculus.GRCm39.103.gff3.gz;

the web links for genome and annotation of Bos indicus are available in https://ftp.ncbi.nlm.nih.gov/genomes/all/GCF/000/247/795/GCF_00024 7795.1_Bos_indicus_1.0/GCF_000247795.1_Bos_indicus_1.0_genomic.fna.gz and https://ftp.ncbi.nlm.nih.gov/genomes/all/GCF/000/247/795/GCF_00024 7795.1_Bos_indicus_1.0/GCF_000247795.1_Bos_indicus_1.0_genomic.gff.gz; and the web links for genome and annotation of Bubalus bubalis are available in https://ftp.ncbi.nlm.nih.gov/genomes/all/GCF/003/121/395/GCF_ 003121395.1_ASM312139v1/GCF_003121395.1_ASM312139v1_genomic.fna. gz and https://ftp.ncbi.nlm.nih.gov/genomes/all/GCF/003/121/395/GCF_ 003121395.1_ASM312139v1/GCF_003121395.1_ASM312139v1_genomic.gff. gz.

The accession numbers listed in Table 1 and Additional file 1 can be referred in the Ensembl database (http://asia.ensembl.org/index.html) and/or NCBI Gene repository (https://www.ncbi.nlm.nih.gov/gene/).

All the other data generated or analyzed during this study are included in this published article and its supplementary information files.

\section{Declarations}

\section{Ethics approval and consent to participate}

The Animal Ethics Committees of Ningxia University approved the experimental design and animal sample collection for the present study (permit number NXUC20200618). We also obtained the written informed consent to participate from the owners and the Ethics Committees of Zerui ecological breeding farm (permit number ZR20200615). And animal experiments were conducted strictly followed the guidelines of the Regulations for the Administration of Affairs Concerning Experimental Animals (Ministry of Science and Technology, China, 2004).

\section{Consent for publication}

Not applicable.

\section{Competing interests}

The authors declare that we have no competing interests.

\section{Author details}

${ }^{1}$ School of Agriculture, Ningxia University, Yinchuan 750021, China. ${ }^{2}$ Key Laboratory of Ruminant Molecular and Cellular Breeding, Ningxia Hui Autonomous Region, Ningxia University, Yinchuan 750021, China. ${ }^{3}$ College of Life Sciences, Xinyang Normal University, Xinyang 464000, Henan, China.

Received: 13 November 2020 Accepted: 27 April 2021

Published online: 12 July 2021

\section{References}

1. Rosen ED, MacDougald OA. Adipocyte differentiation from the inside out. Nat Rev Mol Cell Biol. 2006;7(12):885-96. https://doi.org/10.1038/nrm2066.

2. Gregoire FM, Smas CM, Sul HS. Understanding adipocyte differentiation. Physiol Rev. 1998;78(3):783-809. https://doi.org/10.1152/physrev.1998.78.3. 783.

3. Ntambi JM, Young-Cheul K. Adipocyte differentiation and gene expression. J Nutr. 2000;130(12):3122S-6S. https://doi.org/10.1093/jn/130.12.3122S.

4. Phelps DE, Xiong Y. Regulation of cyclin-dependent kinase 4 during adipogenesis involves switching of cyclin D subunits and concurrent binding of p18INK4c and p27Kip1. Cell Growth Differ. 1998;9(8):595-610.

5. Guo L, Li X, Tang QQ. Transcriptional regulation of adipocyte differentiation: a central role for CCAAT/enhancer-binding protein (C/EBP) beta. J Biol Chem. 2015;290(2):755-61. https://doi.org/10.1074/jbc.R114.619957.

6. Fox KE, Fankell DM, Erickson PF, Majka SM, Crossno JJ, Klemm DJ. Depletion of CAMP-response element-binding protein/ATF1 inhibits adipogenic conversion of 3T3-L1 cells ectopically expressing CCAAT/enhancer-binding protein (C/EBP) alpha, C/EBP beta, or PPAR gamma 2. J Biol Chem. 2006; 281(52):40341-53. https://doi.org/10.1074/jbc.M605077200.

7. Cristancho AG, Lazar MA. Forming functional fat: a growing understanding of adipocyte differentiation. Nat Rev Mol Cell Biol. 2011;12(11):722-34. https://doi.org/10.1038/nrm3198.

8. Liu S, Huang J, Wang $X$, Ma Y. Transcription factors regulate adipocyte differentiation in beef cattle. Anim Genet. 2020;51(3):351-7. https://doi.org/1 0.1111 /age.12931.

9. Horton JD, Shah NA, Warrington JA, Anderson NN, Park SW, Brown MS, et al. Combined analysis of oligonucleotide microarray data from transgenic and knockout mice identifies direct SREBP target genes. Proc Natl Acad Sci U S A. 2003;100(21):12027-32. https://doi.org/10.1073/pnas.1534923100.

10. Malumbres M. Cyclin-dependent kinases. Genome Biol. 2014;15(6):122 https://doi.org/10.1186/gb4184.

11. Hochegger H, Takeda S, Hunt T. Cyclin-dependent kinases and cell-cycle transitions: does one fit all? Nat Rev Mol Cell Biol. 2008;9(11):910-6. https:// doi.org/10.1038/nrm2510.

12. Malumbres M, Harlow E, Hunt T, Hunter T, Lahti JM, Manning G, et al. Cyclin-dependent kinases: a family portrait. Nat Cell Biol. 2009;11(11):1275-6. https://doi.org/10.1038/ncb1109-1275.

13. Malumbres M, Barbacid M. Mammalian cyclin-dependent kinases. Trends Biochem Sci. 2005;30(11):630-41. https://doi.org/10.1016/j.tibs.2005.09.005.

14. Ahonen MA, Haridas P, Mysore R, Wabitsch M, Fischer-Posovszky P. Olkkonen VM: miR-107 inhibits CDK6 expression, differentiation, and lipid storage in human adipocytes. Mol Cell Endocrinol. 2019;479:110-6. https:// doi.org/10.1016/j.mce.2018.09.007.

15. Li X, Kim JW, Gronborg M, Urlaub H, Lane MD, Tang QQ. Role of cdk2 in the sequential phosphorylation/activation of C/EBPbeta during adipocyte differentiation. Proc Natl Acad Sci U S A. 2007;104(28):11597-602. https:// doi.org/10.1073/pnas.0703771104.

16. Lagarrigue S, Lopez-Mejia IC, Denechaud PD, Escote X, Castillo-Armengol J, Jimenez $\mathrm{V}$, et al. CDK4 is an essential insulin effector in adipocytes. J Clin Invest. 2016;126(1):335-48. https://doi.org/10.1172/JCl81480.

17. Abella A, Dubus P, Malumbres M, Rane SG, Kiyokawa H, Sicard A, et al. Cdk4 promotes adipogenesis through PPARgamma activation. Cell Metab. 2005; 2(4):239-49. https://doi.org/10.1016/j.cmet.2005.09.003.

18. Wang H, Liu L, Lin JZ, Aprahamian TR, Farmer SR. Browning of white adipose tissue with Roscovitine induces a distinct population of UCP1(+) 
adipocytes. Cell Metab. 2016;24(6):835-47. https://doi.org/10.1016/j.cmet.201 6.10 .005 .

19. Helenius K, Yang Y, Alasaari J, Makela TP. Mat1 inhibits peroxisome proliferator-activated receptor gamma-mediated adipocyte differentiation. Mol Cell Biol. 2009;29(2):315-23. https://doi.org/10.1128/MCB.00347-08.

20. Zhao X, Feng D, Wang Q, Abdulla A, Xie XJ, Zhou J, et al. Regulation of lipogenesis by cyclin-dependent kinase 8-mediated control of SREBP-1. J Clin Invest. 2012;122(7):2417-27. https://doi.org/10.1172/JCl61462.

21. Vallbracht M, Fuchs W, Klupp BG, Mettenleiter TC. Functional relevance of the transmembrane domain and cytoplasmic tail of the pseudorabies virus glycoprotein h for membrane fusion. J Virol. 2018;92(12):e00376-18. https:// doi.org/10.1128/JVI.00376-18.

22. Ota M, Kinoshita K, Nishikawa K. Prediction of catalytic residues in enzymes based on known tertiary structure, stability profile, and sequence conservation. J Mol Biol. 2003;327(5):1053-64. https://doi.org/10.1016/ S0022-2836(03)00207-9.

23. Van Montfort RL, Pijning T, Kalk KH, Hangyi I, Kouwijzer ML, Robillard GT, et al. The structure of the Escherichia coli phosphotransferase IIAmannitol reveals a novel fold with two conformations of the active site. Structure. 1998;6(3):377-88. https://doi.org/10.1016/S0969-2126(98)00039-2.

24. Song L, Wu S, Tsang A. Phylogenetic analysis of protein family. Methods Mol Biol. 1775;2018:267-75. https://doi.org/10.1007/978-1-4939-7804-5_21.

25. Xie T, Chen C, Li C, Liu J, Liu C, He Y. Genome-wide investigation of WRKY gene family in pineapple: evolution and expression profiles during development and stress. BMC Genomics. 2018;19(1):490. https://doi.org/1 0.1186/s12864-018-4880-x.

26. Gerdol M, Greco S, Pallavicini A. Extensive tandem duplication events drive the expansion of the clq-domain-containing gene family in bivalves. Mar Drugs. 2019;17(10):583.

27. Krsticevic FJ, Arce DP, Ezpeleta J, Tapia E. Tandem duplication events in the expansion of the small heat shock protein gene family in Solanum lycopersicum (cv. Heinz 1706). G3 (Bethesda). 2016;6(10):3027-34. https:// doi.org/10.1534/g3.116.032045.

28. Zimin AV, Delcher AL, Florea L, Kelley DR, Schatz MC, Puiu D, et al. A wholegenome assembly of the domestic cow, Bos taurus. Genome Biol. 2009; 10(4):R42. https://doi.org/10.1186/gb-2009-10-4-r42.

29. Hu Q, Ma T, Wang K, Xu T, Liu J, Qiu Q. The yak genome database: an integrative database for studying yak biology and high-altitude adaption. BMC Genomics. 2012;13(1):600. https://doi.org/10.1186/1471-2164-13-600.

30. Liu J, Wang Z, Li J, Li H, Yang L. Genome-wide identification of Diacylglycerol Acyltransferases (DGAT) family genes influencing Milk production in Buffalo. BMC Genet. 2020;21(1):26. https://doi.org/10.1186/s12863-020-0832-y.

31. Weckselblatt $B$, Rudd MK. Human structural variation: mechanisms of chromosome rearrangements. Trends Genet. 2015;31(10):587-99. https://doi. org/10.1016/j.tig.2015.05.010.

32. Parua PK, Kalan S, Benjamin B, Sanso M, Fisher RP. Distinct Cdk9phosphatase switches act at the beginning and end of elongation by RNA polymerase II. Nat Commun. 2020;11(1):4338. https://doi.org/10.1038/s414 67-020-18173-6

33. lankova I, Petersen RK, Annicotte JS, Chavey C, Hansen JB, Kratchmarova I, et al. Peroxisome proliferator-activated receptor gamma recruits the positive transcription elongation factor b complex to activate transcription and promote adipogenesis. Mol Endocrinol. 2006;20(7):1494-505. https://doi. org/10.1210/me.2005-0222.

34. Mu RF, Niu YF, Wang Q, Zhou HM, Hu J, Qin WY, et al. Eriocalyxin B inhibits Adipogenesis in 3T3-L1 adipocytes by cell cycle arrest. Nat Prod Bioprospect. 2020;10(3):131-40. https://doi.org/10.1007/s13659-02 0-00240-6.

35. Marquez MP, Alencastro F, Madrigal A, Jimenez JL, Blanco G, Gureghian A, et al. The role of cellular proliferation in Adipogenic differentiation of human adipose tissue-derived Mesenchymal stem cells. Stem Cells Dev. 2017;26(21):1578-95. https://doi.org/10.1089/scd.2017.0071.

36. Stetkova M, Growkova K, Fojtik P, Valcikova B, Palusova V, Verlande A, et al. CDK9 activity is critical for maintaining MDM4 overexpression in tumor cells. Cell Death Dis. 2020;11(9):754. https://doi.org/10.1038/s4141 9-020-02971-3.

37. Mbonye U, Wang B, Gokulrangan G, Shi W, Yang S, Karn J. Cyclindependent kinase 7 (CDK7)-mediated phosphorylation of the CDK9 activation loop promotes P-TEFb assembly with tat and proviral HIV reactivation. J Biol Chem. 2018;293(26):10009-25. https://doi.org/10.1074/jbc. RA117.001347.
38. Shannon P, Markiel A, Ozier O, Baliga NS, Wang JT, Ramage D, et al. Cytoscape: a software environment for integrated models of biomolecular interaction networks. Genome Res. 2003;13(11):2498-504. https://doi.org/1 $0.1101 /$ gr. 1239303

39. Hallenborg P, Siersbaek M, Barrio-Hernandez I, Nielsen R, Kristiansen K, Mandrup S, et al. MDM2 facilitates adipocyte differentiation through CRTCmediated activation of STAT3. Cell Death Dis. 2016;7(6):e2289. https://doi. org/10.1038/cddis.2016.188.

40. Li Y, He L, Li J, Chen J, Liu C. Genome-wide identification, characterization, and expression profiling of the legume BZR transcription factor gene family. Front Plant Sci. 2018;9:1332. https://doi.org/10.3389/fpls.2018.01332.

41. El-Gebali S, Mistry J, Bateman A, Eddy SR, Luciani A, Potter SC, et al. The Pfam protein families database in 2019. Nucleic Acids Res. 2019;47(D1): D427-32. https://doi.org/10.1093/nar/gky995.

42. Potter SC, Luciani A, Eddy SR, Park Y, Lopez R, Finn RD. HMMER web server: 2018 update. Nucleic Acids Res. 2018;46(W1):W200-4. https://doi.org/10.1 093/nar/gky448

43. Lu S, Wang J, Chitsaz F, Derbyshire MK, Geer RC, Gonzales NR, et al. CDD/ SPARCLE: the conserved domain database in 2020. Nucleic Acids Res. 2020; 48(D1):D265-8. https://doi.org/10.1093/nar/gkz991.

44. Artimo P, Jonnalagedda M, Arnold K, Baratin D, Csardi G, de Castro E, et al. ExPASy: SIB bioinformatics resource portal. Nucl Acids Res. 2012;40(Web Server issue):W597-603. https://doi.org/10.1093/nar/gks400.

45. Kumar S, Stecher G, Tamura K. MEGA7: molecular evolutionary genetics analysis version 7.0 for bigger datasets. Mol Biol Evol. 2016;33(7):1870-4. https://doi.org/10.1093/molbev/msw054.

46. Bailey TL, Johnson J, Grant CE, Noble WS. The MEME suite. Nucleic Acids Res. 2015;43(W1):W39-49. https://doi.org/10.1093/nar/gkv416.

47. Chen $\mathrm{C}$, Chen H, Zhang Y, Thomas HR, Frank MH, He Y, et al. TBtools: an integrative toolkit developed for interactive analyses of big biological data. Mol Plant. 2020;13(8):1194-202. https://doi.org/10.1016/j.molp.2020.06.009.

48. Wang Y, Tang H, Debarry JD, Tan X, Li J, Wang X, et al. MCScanX: a toolkit for detection and evolutionary analysis of gene synteny and collinearity. Nucleic Acids Res. 2012;40(7):e49. https://doi.org/10.1093/nar/gkr1293.

49. Cai H, Li M, Sun X, Plath M, Li C, Lan X, et al. Global Transcriptome analysis during Adipogenic differentiation and involvement of Transthyretin gene in Adipogenesis in cattle. Front Genet. 2018;9:463. https://doi.org/10.3389/ fgene.2018.00463.

50. Wingett SW, Andrews S. FastQ Screen: a tool for multi-genome mapping and quality control. F1000Res. 2018;(7):1338. https://doi.org/10.12688/f1 000research.15931.2.

51. Utturkar S, Dassanayake A, Nagaraju S, Brown SD. Bacterial differential expression analysis methods. Methods Mol Biol. 2020;2096:89-112. https:// doi.org/10.1007/978-1-0716-0195-2_8.

52. Dobin A, Gingeras TR. Mapping RNA-seq reads with STAR. Curr Protoc Bioinformatics. 2015;51:11.14.1-11.14.19. https://doi.org/10.1002/0471250953. bi1114s51.

53. Li B, Dewey CN. RSEM: accurate transcript quantification from RNA-Seq data with or without a reference genome. BMC Bioinform. 2011;12(1):323. https:// doi.org/10.1186/1471-2105-12-323.

54. Liao Y, Smyth GK. Shi W: featureCounts: an efficient general purpose program for assigning sequence reads to genomic features. Bioinformatics. 2014;30(7):923-30. https://doi.org/10.1093/bioinformatics/btt656.

55. Law CW, Alhamdoosh M, Su S, Dong X, Tian L, Smyth GK, et al. RNA-seq analysis is easy as 1-2-3 with limma, Glimma and edgeR. F1000Res. 2016;5: ISCB Comm J-1408. https://doi.org/10.12688/f1000research.9005.3.

56. Chen L, Qiu Q, Jiang Y, Wang K, Lin Z, Li Z, et al. Large-scale ruminant genome sequencing provides insights into their evolution and distinct traits. Science. 2019;364(6446):eaav6202. https://doi.org/10.1126/science.aav6202.

57. Galili T, O'Callaghan A, Sidi J, Sievert C. Heatmaply: an R package for creating interactive cluster heatmaps for online publishing. Bioinformatics. 2018;34(9):1600-2. https://doi.org/10.1093/bioinformatics/btx657.

58. Huang J, Zheng Q, Wang S, Wei X, Li F, Ma Y. High-throughput rna sequencing reveals ndufc2-as Incrna promotes adipogenic differentiation in chinese buffalo (Bubalus bubalis L). Genes (Basel). 2019;10(9):689. https://doi. org/10.3390/genes10090689.

59. Wang $Y$, Goulart RA, Pantanowitz L. Oil red $O$ staining in cytopathology. Diagn Cytopathol. 2011;39(4):272-3. https://doi.org/10.1 002/dc.21390.

60. Adnan M, Morton G, Hadi S. Analysis of rpoS and bolA gene expression under various stress-induced environments in planktonic and biofilm phase 
using 2(-DeltaDeltaCT) method. Mol Cell Biochem. 2011;357(1-2):275-82. https://doi.org/10.1007/s11010-011-0898-y.

61. Arocho A, Chen B, Ladanyi M, Pan Q. Validation of the 2-DeltaDeltaCt calculation as an alternate method of data analysis for quantitative PCR of BCR-ABL P210 transcripts. Diagn Mol Pathol. 2006;15(1):56-61. https://doi. org/10.1097/00019606-200603000-00009.

\section{Publisher's Note}

Springer Nature remains neutral with regard to jurisdictional claims in published maps and institutional affiliations.

Ready to submit your research? Choose BMC and benefit from:

- fast, convenient online submission

- thorough peer review by experienced researchers in your field

- rapid publication on acceptance

- support for research data, including large and complex data types

- gold Open Access which fosters wider collaboration and increased citations

- maximum visibility for your research: over $100 \mathrm{M}$ website views per year

At BMC, research is always in progress.

Learn more biomedcentral.com/submissions 